\section{(1) \\ CrossMark}

\title{
A new two-step algorithm for the treatment of COPD
}

\author{
To the Editor:
}

The pharmacological treatment of chronic obstructive pulmonary disease (COPD) is quite uniformly based on long-acting bronchodilators (LABD), either a long-acting muscarinic antagonist (LAMA) or a long-acting $\beta$-agonist (LABA), and inhaled corticosteroids (ICS). Moreover, most patients are eventually treated with a combination of all three (LAMA/LABA/ICS or "triple therapy") irrespective of their severity and level of risk. Furthermore, a large proportion of patients receive triple therapy as the initial treatment [1]. We propose a new simple treatment algorithm based on the severity of symptoms and phenotypes or treatable traits (figure 1).

Once the diagnosis of COPD has been confirmed by spirometry, the first decision is whether to prescribe either one LABD or dual bronchodilation (LABA/LAMA) therapy. This decision should be based on the level of dyspnoea (as measured by the modified Medical Research Council dyspnoea scale; mMRC) and the frequency of exacerbations. Patients with an mMRC score of one and zero or one exacerbation during the previous year may start treatment with a LAMA, whereas patients with either an mMRC score higher than one or with more than one exacerbation in the previous year should start with a LABA/LAMA combination. It is very unusual for a patient to be diagnosed with COPD at a stage of the disease so severe that it would require more intensive initial therapy. In any case, even under such circumstances, it seems reasonable to initiate treatment with a LABA/LAMA combination and observe the response before introducing other second-line therapies. We recommend initial therapy with a LAMA because, despite the similar bronchodilatory efficacy of LABAs, LAMAs provide superior protection against exacerbations [2]. However, LABAs can be an option particularly if there is no history of previous exacerbations.

The selection of initial therapy with a LABA/LAMA combination for patients with more intense dyspnoea (mMRC 2+) is based on the better results obtained with this combination versus bronchodilator monotherapy in patients with an mMRC dyspnoea score higher than one in terms of lung function, symptoms and quality of life [3]. Furthermore, this combination is preferred over an LABA/ICS combination as the initial therapy because it provides significantly better lung function improvement without the risk of side effects associated with long-term use of ICS, such as pneumonia [4]. It should be noted, however, that information about the long-term safety of LAMA/LABA combinations is still lacking. The recommendation of initial therapy with a LABA/LAMA combination in patients with more than one exacerbation during the previous year is justified by its higher efficacy versus LAMA monotherapy in the prevention of exacerbations [5] and especially for its superiority versus LABA/ICS combinations in the prevention of exacerbations [6].

After initiation of therapy with a LAMA, a patient with mild COPD may remain stable with improved dyspnoea and no exacerbations. In this case treatment must be continued; however, if dyspnoea does not improve or deteriorates over time, or if exacerbations become frequent, then treatment should be stepped-up to a LABA/LAMA combination. On treatment with a LAMA/LABA combination, either as initial therapy or after step-up, patients may remain stable and must continue with the same therapy. This includes the presence of one ambulatory exacerbation a year. Obviously this is an arbitrary threshold but we believe that presenting one mild or moderate exacerbation a year should not be a reason to add a second-line drug (usually an anti-inflammatory) due to the associated risk of side effects. However, this decision should be made by the physician on a case by case basis taking into account the balance between the expected benefits and risks.

@ERSpublications

Treatment of COPD can be simplified with a two-step algorithm according to severity and phenotype http://ow.ly/iJUN306DEHU

Cite this article as: Miravitlles M, Anzueto A. A new two-step algorithm for the treatment of COPD. Eur Respir J 2017; 49: 1602200 [https://doi.org/10.1183/13993003.02200-2016]. 


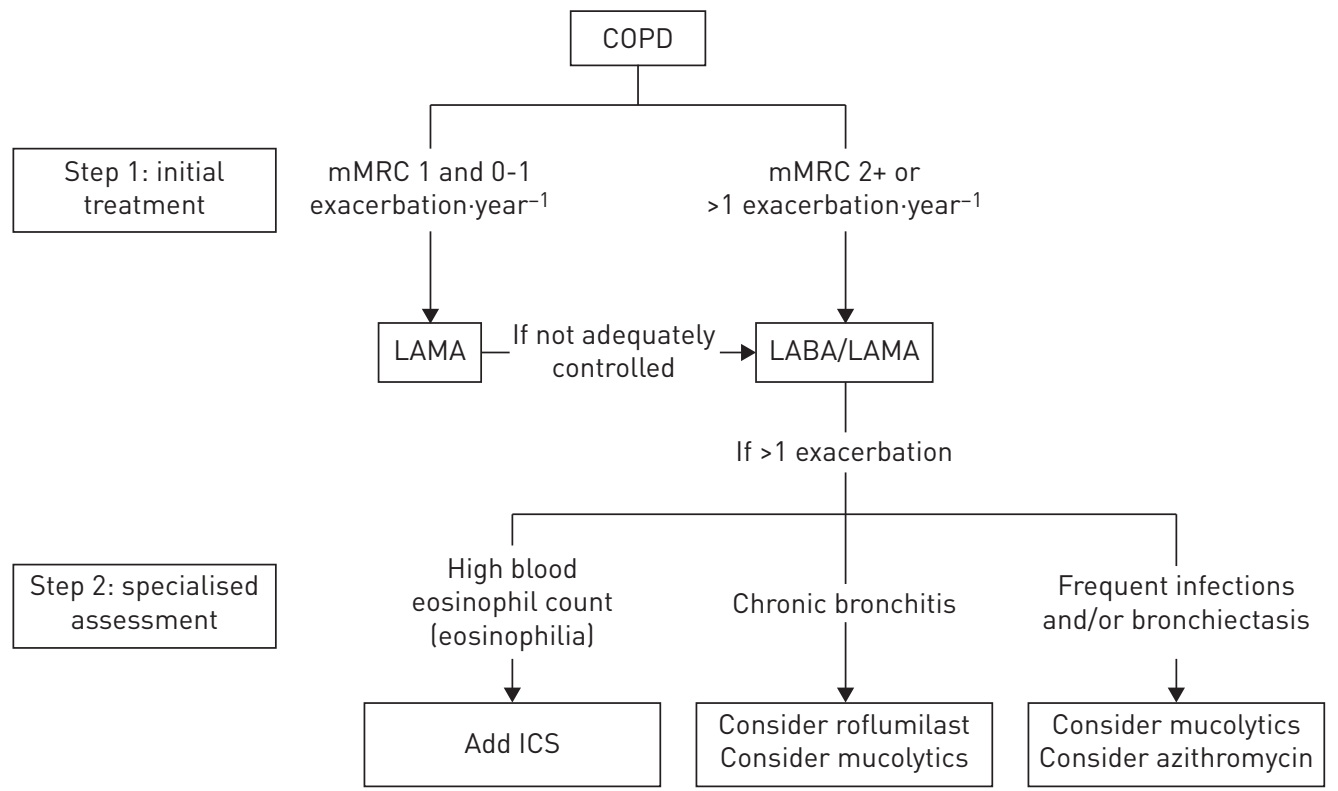

FIGURE 1 A two-step algorithm for the treatment of chronic obstructive pulmonary disease (COPD). mMRC: modified Medical Research Council dyspnoea scale; LABA: long-acting $\beta$-agonist; LAMA: long-acting muscarinic antagonist; ICS: inhaled corticosteroids.

If there is deterioration in symptoms, or if the patient experiences more than one exacerbation in a year or requires hospitalisation for an exacerbation while on LABA/LAMA therapy, a step-up in treatment should be considered. These patients are very challenging and should be treated by experienced professionals in settings in which the appropriate diagnostic tests are available. The reason for this is that no single approach is valid for all patients in this category [7].

The most frequent complaint among COPD patients is an increase in the number of exacerbations or their severity, in which case a specific therapeutic pharmacological approach must be established based on the phenotype. ICS are the most extensively studied and the most widely used anti-inflammatory drugs for COPD. They are indicated for the prevention of exacerbation in combination with bronchodilators. Recent data suggest that the preventive effects of ICS on COPD exacerbation are higher in patients with an eosinophilic (i.e. Th2 type) inflammatory profile, which can be demonstrated by elevated concentrations of eosinophils in peripheral blood [8], although these data must be confirmed in prospective studies. There is also consensus that patients with COPD and characteristics of asthma (asthma-COPD overlap (ACO)) should be treated with ICS in addition to bronchodilators [9]. In contrast, the insignificantly small efficacy levels of ICS on top of bronchodilators in patients with low levels of blood eosinophils, together with the risk of side-effects associated with their long-term use, advise against their indication in this context.

However, ICS are not the only option after dual bronchodilators. Roflumilast is an effective oral anti-inflammatory drug that has been demonstrated to reduce exacerbation in patients with severe COPD and chronic bronchitis when used in combination with a LABD, a LABA/ICS combination, or even in addition to triple therapy [10]. Unfortunately, its use is associated with frequent adverse events such as weight loss, nausea and diarrhoea that may limit the tolerability of the drug.

Although the Broncus trial with $\mathrm{N}$-acetylcystein (NAC) was negative, recent studies performed in Asia have shown significantly reduced exacerbations with mucolytics such as carbocystein or NAC at high doses (600 mg twice daily), particularly in patients at high risk as defined in the Global Initiative for Chronic Obstructive Lung Disease (GOLD) strategy (i.e. those with forced expiratory volume in $1 \mathrm{~s}$ (FEV 1 ) $<50 \%$ of predicted, or with two or more frequent exacerbations in the previous year, or both) [11].

The use of macrolides has been associated with a variety of effects beyond their anti-infective activity. The most widely used macrolide is azithromycin and several trials have demonstrated the efficacy of its long-term administration in the prevention of exacerbations [12], in particular in patients with severe COPD who suffer frequent exacerbations (more than three episodes a year) despite optimal-inhalation therapy [13]. However, the long-term use of macrolides may be associated with important side-effects and the risk of developing of bacterial resistance [12]. 
Recently, CABrera et al. [14] demonstrated that a simple algorithm based on dyspnoea and the frequency of exacerbations classified patients in a similar manner to the new GOLD strategy; however, they also used the frequency of exacerbations as an indicator for the introduction of ICS. Interestingly, they used the same cut-off point as in our algorithm (that of an mMRC score of 2+) to recommend dual bronchodilators. It is of note that their algorithm was published before the results of the FLAME trial became available [6]. The algorithm published by COOPER and BARJAKTAREvic [15] is very similar to our own proposal and suggests the use of dual bronchodilation in all cases before other drugs are considered. Furthermore, they propose a phenotype-oriented therapy in addition to a LABA/LAMA combination for the more severe cases, such as those patients who suffer exacerbations despite maximal bronchodilation. We have extended this algorithm by proposing the use of phenotype-oriented therapy for those who exacerbate on top of dual bronchodilators, based on a previous algorithm developed exclusively for exacerbators [7]. This proposal can also be considered as an approach based on phenotypes because it selects the different treatments based on patient characteristics that identify responders to the drugs.

Marc Miravitlles $^{1}$ and Antonio Anzueto ${ }^{2,3}$

${ }^{1}$ Dept of Pneumology, Hospital Universitari Vall d'Hebron, Centro de Investigación Biomédica en Red Enfermedades Respiratorias (CIBERES), Barcelona, Spain. ${ }^{2}$ Division of Pulmonary Diseases and Critical Care Medicine, School of Medicine, The University of Texas Health Science Center, San Antonio, TX, USA. ${ }^{3}$ Audie L. Murphy Memorial VA Hospital, South Texas Veterans Health Care System, San Antonio, TX, USA.

Correspondence: Marc Miravitlles, Dept of Pneumology, Hospital Universitari Vall d'Hebron, P. Vall d'Hebron 119-129, ES-08035, Barcelona, Spain. E-mail: mmiravitlles@vhebron.net

Received: Sept 212016 | Accepted after revision: Nov 202016

Conflict of interest: Disclosures can be found alongside this article at erj.ersjournals.com

\section{References}

1 Barrecheguren M, Monteagudo M, Ferrer J, et al. Treatment patterns in COPD patients newly diagnosed in primary care. A population-based study. Respir Med 2016; 111: 47-53.

2 Vogelmeier C, Hederer B, Glaab T, et al. Tiotropium versus salmeterol for the prevention of exacerbations of COPD. N Engl J Med 2011; 364: 1093-1103.

3 Buhl R, Maltais F, Abrahams R, et al. Tiotropium and olodaterol fixed-dose combination versus monocomponents in COPD (GOLD 2-4). Eur Respir J 2015; 45: 969-979.

4 Vogelmeier C, Paggiaro PL, Dorca J, et al. Efficacy and safety of aclidinium/formoterol versus salmeterol/ fluticasone: a phase 3 COPD study. Eur Respir J 2016; 48: 1030-1039 [DOI: 10.1183/13993003.00216-2016].

5 Wedzicha JA, Decramer M, Ficker JH, et al. Analysis of chronic obstructive pulmonar disease exacerbations with the dual bronchodilator QVA149 compared with glycopyrronium and tiotropium (SPARK): a randomised, double-blind, parallel-group study. Lancet Respir Med 2013; 1: 199-209.

6 Wedzicha JA, Banerji D, Chapman KR, et al. Indacaterol-Glycopyrronium versus Salmeterol-Fluticasone for COPD. N Engl J Med 2016; 374: 2222-2234.

7 Miravitlles M, D'Urzo A, Singh D, et al. Pharmacological strategies to reduce exacerbation risk in COPD: a narrative review. Respir Res 2016; 17: 112.

8 Pavord ID, Lettis S, Locantore N, et al. Blood eosinophils and inhaled corticosteroid/long-acting $\beta$-2 agonist efficacy in COPD. Thorax 2016; 71: 118-125.

9 Sin DD, Miravitlles M, Mannino DM, et al. What is asthma-COPD overlap syndrome (ACOS)? Towards a consensus definition from a roundtable discussion. Eur Respir J 2016; 48: 664-673.

10 Martinez FJ, Calverley PM, Goehring UM, et al. Effect of roflumilast on exacerbations in patients with severe chronic obstructive pulmonary disease uncontrolled by combination therapy (REACT): a multicentre randomised controlled trial. Lancet 2015; 385: 857-866.

11 Tse HN, Raiteri L, Wong KY, et al. Benefits of high-dose N-acetylcysteine to exacerbation-prone patients with COPD. Chest 2014; 146: 611-623.

12 Miravitlles M, Anzueto A. Antibiotics for acute and chronic respiratory infection in patients with chronic obstructive pulmonary disease. Am J Respir Crit Care Med 2013; 188: 1052-1057.

13 Uzun S, Djamin RS, Kluytmans JA, et al. Azithromycin maintenance treatment in patients with frequent exacerbations of chronic obstructive pulmonary disease (COLUMBUS): a randomised, double-blind, placebo-controlled trial. Lancet Respir Med 2014; 2: 361-368.

14 Cabrera C, Casanova C, Martín Y, et al. Agreement between a simple dyspnea-guided treatment algorithm for stable COPD and the GOLD guidelines: a pilot study. Int J Chron Obst Pulm Dis 2016; 11: 1217-1222.

15 Cooper CB, Barjaktarevic I. A new algorithm for the management of COPD. Lancet Respir Med 2015; 3: 266-268. 